\title{
Surveillance Impediments: \\ Article Recognizing Obduracy with the Deployment of Hospital Information Systems
}

\section{Torin Monahan}

Vanderbilt University, USA. torin.monahan@,vanderbilt.edu

\author{
Jill A. Fisher \\ Vanderbilt University, USA. jill.fisher@,vanderbilt.edu
}

\begin{abstract}
Although the field of Surveillance Studies privileges detailed accounts of how and when surveillance occurs, it is also important to remain open to instances of aborted or failed surveillance and manifold impediments to surveillance. It is vital for researchers to document and theorize absence in order to better understand and perhaps mitigate the presence of egregious forms of surveillance. To this end, in this paper we discuss some of the many forms of obduracy that confront surveillance-capable information systems in hospitals. We draw upon primary data from three years of field research on the implementation and use of tracking and identification systems in U.S. hospitals to show that many of the control functions of hospital information systems are attenuated by individual resistance but also by a host of technical, material, financial, and cultural constraints.
\end{abstract}

The field of Surveillance Studies often lures scholars into deterministic readings of new technologies. After all, "the surveillance society" is a powerful concept that reorients the perceptions of scholars to see all information and communication technologies (ICTs) as having the potential, even the valence, for cultivating surveillant practices and structuring surveillant relationships (Lyon 2001; Monahan 2010; Murakami Wood et al. 2006). This is a theoretical position that is grounded in fact, in observations of mission creep that can and does occur with the deployment of technological systems. Yet potentialities and probabilities are not the same thing as inevitabilities. And the field may be guilty of being too alarmist or "paranoid" because its scholars, including us, are often committed to discovering surveillance in every domain on which they set their sights (Holm 2009; Lyon 1994). It is also important, however, to remain open to instances of non-surveillance, aborted or failed surveillance, or manifold impediments to surveillance. In a word, it is vital for researchers to document and theorize absence in order to better understand and perhaps mitigate the presence of egregious forms of surveillance.

In this paper, we discuss some of the many forms of obduracy that confront surveillance-capable information systems in hospitals. We draw upon primary data from three years of field research on the implementation and use of tracking and identification systems in U.S. hospitals to show that many of the control functions of hospital information systems are attenuated by individual resistance but also by a host of organizational and infrastructural constraints. While it is the case that surveillance functions are enabled

Monahan, T. and J.A. Fisher. 2011 . Surveillance Impediments: Recognizing Obduracy with the Deployment of Hospital Information Systems. Surveillance \& Society 9(1/2): 1-16.

http://www.surveillance-and-society.org I ISSN: 1477-7487

(C) The authors, 2011 | Licensed to the Surveillance Studies Network under a Creative Commons Attribution Non-Commercial No Derivatives license. 
by hospital information systems, impediments to surveillance are significant and may often be explained more precisely as the result of institutional and infrastructural inertia than of organized or concerted opposition. After providing some background for our study, this paper sketches out some forms of hospital surveillance that we were able to document and then describes and analyzes four areas of obduracy facing such systems: technical, material, financial, and cultural.

\section{Background and Methods}

The data presented here are part of a study that investigated social and ethical concerns associated with hospital tracking and identification systems, which are also known as "real-time location systems" (RTLS). Such systems can be used to track hospital inventory, staff, or patients, ostensibly with the aim of managing assets, people, and practices in more efficient and effective ways (Monahan and Fisher 2008; Fisher 2006). For instance, hospital administrators can deploy RTLS to assist with locating medical equipment, such as intravenous pumps or specialized beds, whether for purposes of providing immediate patient care, conducting routine scheduled maintenance, preventing theft or loss, reducing redundant inventory, or calculating fees due for rented equipment. Staff can be tracked to better understand and streamline "workflow," automatically assign individual personnel to individual patients for care provision, ascertain who is closest to a person in need, or discipline those who take too frequent breaks or perform their jobs sub-optimally. Patients can be tagged and monitored to verify their identities before medical procedures, locate them if they get "lost" in a hospital, send alerts to staff if they fall, or allow for "dynamic associations" whereby patients can be billed automatically when they come in contact with any devices, drugs, or personnel. Obviously, most hospitals have procedures in place to perform almost all of these functions without the assistance of RTLS. Nonetheless, a combination of technological imperatives, audit cultures, entrepreneurial vendors, and government incentives is currently driving the adoption of these and other health information technology (HIT) systems in hospitals (Blumenthal and Glaser 2007; Fisher and Monahan 2011; Lohr 2009; Thuemmler et al. 2009; Winthereik, van der Ploeg, and Berg 2007).

A disparate array of technologies comprises the field of RTLS. Basically these systems use electronic "tags" that send out signals to "detectors" that are installed in hospital buildings. The tags can communicate via radio-frequency identification (RFID), which is most common, or through ultrasound, infrared, or other signals, across a variety of network protocols. The tags can be affixed to items, embedded in identification cards, or placed on identification armbands, for example. Additionally, most tags being used in hospital contexts are "active" ones that contain a small battery that needs to be replaced periodically. RTLS also typically require infrastructural alterations, such as stringing network cable, installing detectors, adding electrical conduit, and finding room for additional data servers and computer terminals. Finally, software programs are needed to translate the signals received by the detectors into data and provide mechanisms and interfaces for organizing and acting upon those data, preferably in a way that is interoperable with other data-management or patient-care systems.

We conceive of technological systems as being far more than just artifacts, wires, and software. Technologies acquire meaning through social practice in specific organizational contexts; they draw upon and feed the political economy, further situating hospitals within larger macrostructural relations; they are both socially constructed and social shaping, such that they assert agential force upon the roles and responsibilities of healthcare workers and consumers. ${ }^{1}$ Therefore, for the purposes of this project we were especially interested in attending to how people talk about RTLS, what meanings they ascribe to them, and how they do or don't incorporate them into their lives. Moreover, given the clear surveillance

\footnotetext{
1 This orientation to technological systems obviously draws upon insights from the field of science and technology studies. See, for example, Berg (1997), Bijker and Law (1992), Bowker and Star (1999), Mesman (2008), Oudshoorn (2007), and Winner (1986).
} 
overtones of real-time monitoring, particularly of individuals, we were interested in assessing the modes of surveillance engendered by RTLS. Our primary research questions were (1) What effects do real-time location systems have upon organizational roles and relations? and (2) What are the surveillance potentials of real-time location systems? The focus of these questions included documentable practices and policies as well as hospital staff member's perceptions of the changes brought about by RTLS.

The research methods for our project were observation and semi-structured interviews with personnel at hospitals that have implemented or piloted systems to identify or track equipment, staff, and/or patients. The project included 23 U.S. hospitals that were selected based on their use of a qualifying system (as identified through personal contacts, press releases, or media coverage) and their receptivity to participating in the research. One or both authors visited each hospital, were given demonstrations of the systems, and observed the systems in use. In addition, we conducted $80 \mathrm{semi}$-structured interviews with hospital staff, including physicians, nurses, administrators, information technologists, and biomedical engineers, as well as vendors involved with these systems. Interviewees were recruited from hospital employees who make decisions about or are targeted users of the systems. The identities of hospital sites and personnel were given confidentiality in the study, and all participants provided informed consent. We then analyzed all transcribed interviews and observational notes to identify core themes, such as key concerns associated with these systems. One dominant theme was obstacles to the successful deployment of these systems, and especially to the use of the systems for any overt surveillance purposes.

\section{Surveillance Capabilities and Potentials}

Although the primary focus of this paper is on factors contributing to the absence of surveillance, it is important to acknowledge the surveillance capabilities and potentials of RTLS and similar systems. Our research did uncover several troubling instances of surveillance and discipline of hospital staff as well as indications of where surveillance applications could flourish, or creep, in the future.

The ability to locate people in space and real time is enticing for individuals operating in the complex and oftentimes chaotic worlds of hospitals. As one resident physician communicated about the system at his hospital:

Sometimes it's useful for finding where my nurse is. I've noticed there's this system where oh, I can look up where my nurses are. Oh, I can look up where the cart is. So now I'm learning a little bit more about it, like no one really explains all that, but one time I found my attending [physician] sleeping in the pod. It was late at night and he was getting sick or something, and I was like, "Where's my attending?" I looked on there and he was in the pod that was shut down taking a nap because he was so exhausted. So I was like, "Oh, he's over in B-10!" So sometimes it's useful for that.

While locating people can be useful, it is not always benign. RTLS apparently invite discipline if people are not where one thinks they should be or where they say that they are. At industry conferences and in conversations with hospital administrators, we heard of a few instances (at different hospitals) where individuals were fired because their accounts of where they were differed from the evidence generated by the locational systems. For example, one hospital piloting RTLS did not provide much information about the capabilities of the technology to the orderlies who were asked to wear the sensors. At one point, an administrator monitoring the location of the orderlies on a computer interface saw that two orderlies were standing on the loading dock. The administrator contacted one of the orderlies on his walkie-talkie to inquire about his location, and the orderly gave a false account of his activities. Next, the administrator 
contacted the second orderly who provided a different bogus report of his whereabouts. The administrator then walked down to the loading dock to "catch" the two orderlies and fired them both on the spot. ${ }^{2}$

Hospital administrators have interest in monitoring employees for a range of reasons, so the type of personnel who are monitored varies by hospital. Employees with less status and institutional power tend to be the ones subjected to locational tracking. Physicians are monitored directly too, but less often than other staff and with less serious sanctions for undesirable performance. For instance, some hospitals use RTLS to track how long physicians spend with patients, while other hospitals integrate RTLS with different reporting mechanisms to try to pressure physicians into practicing in more cost-effective ways, especially in departments of emergency medicine. One administrator explained:

We monitor all the activity of all our docs. We give them reports every month on their acuity, their time to disposition, the number of tests per doctor per diagnosis, their efficiency in terms of what we pay through the department, and that's all recorded. And your abilities, your bonuses, all that information is used as a 360 [-degree] view of you as an emergency physician in the department. But if you're admitting twenty-two percent or twenty-three percent, twenty-four percent, then you're being very conservative [with your patients] and you should be more tight with your admissions because you're actually losing [the hospital] money... The chairman would say, "You know, the last couple months you've been admitting a lot more percentiles. Just try to bring it down a little bit."

These reporting mechanisms can work through a type of public shaming: monthly reports identify physicians' performance scores alongside their names. Physicians tend to be rewarded rather than punished for their performance using a series of incentive-based processes (often financial) rather than disciplinary ones.

For nurses and other hospital staff, at least at present, RTLS-facilitated surveillance manifests indirectly more often than through direct scrutiny. For instance, administrators can use RTLS data to chastise nurses who are perceived as not furthering hospitals' goals of improving patient throughput. Nurses understandably try to control their own workloads, reduce stress, and increase quality of care by pacing the number of patients for whom they are responsible. Specifically, hospitals tend to rely upon nurses to signal when a patient leaves the hospital so that the patient's room - or bed - can be cleaned, thereby freeing up that space for a new patient. A new patient moving into that room means an increase in labor for the nurse(s) assigned to that patient, so nurses might delay reporting the discharge until they are caught up with their work or they are changing shifts. If a patient is assigned an RTLS tag upon admission, however, bed management can be automated: when a patient's RTLS tag leaves the hospital, presumably with the patient, the system assumes that the patient has been discharged and his or her room is now signaled as ready to be cleaned. In response to these automated mechanisms for increasing throughput, sometimes nurses will find ways to circumvent the systems. One hospital IT worker explained:

Nurses don't particularly care to discharge a patient and admit another patient anywhere near their shift time, end of shift, so they found some "workarounds": cutting the actual location tag off and throwing it in the trash can cause that location to continue to be there. You know that patient is still showing [on the system as being] in their room when in fact they've left the hospital.

In order to detect this type of nurses' resistance to RTLS, administrators can compare patient discharge times as indicated by the bed-management system and the electronic medical record system. One hospital

\footnotetext{
2 A technology vendor recounted this story during a Q\&A session following a presentation by the hospital administrator at the 2008 Healthcare Information and Management Systems Society (HIMSS) conference in Orlando, FL.
} 
we visited was actively monitoring nurses in this way, and an administrator told us, "If there's a big discrepancy there, then we contact the manager and they go ruffle some feathers." ${ }^{3}$

Employees in hospitals may experience heightened surveillance by means of RTLS, especially surveillance of their performance and behavior, which is in keeping with findings of workplace surveillance in other domains (Ball 2005; Regan 1998; Staples 2000). ${ }^{4}$ All in all, though, our research discovered few examples of surveillance, relative to data about other concerns or problems with locational systems in hospitals. The potential for mission creep is certainly there, especially when clear policies are not delineated in advance and stakeholders are not involved in - or even informed of - decisions (Fisher and Monahan 2008). Still, what was far more striking were the many impediments to these systems and their surveillance capabilities.

\section{Technical Constraints}

Perhaps the biggest obstacle to surveillance with hospital RTLS is that the systems, by and large, do not work. That is to say, at least at present, they do not work with a sufficient degree of accuracy, consistency, or speed to locate people or objects reliably in the complex organizational spaces of most hospitals. Whereas RFID systems have achieved celebrated success for their efficacy in tracking items on assembly lines or through other linear and tightly controlled distribution channels, such as the systems for delivering pallets of inventory to Wal-Mart stores, hospitals are far too nonlinear, unpredictable, and chaotic for locational systems to achieve similar levels of success in these environments.

With radio-frequency systems in particular, the signals bleed through walls and floors, such that locational readings are usually inaccurate and sometimes grossly so. At just about every hospital site we visited, people had complaints about the lack of room-level accuracy with these systems, but a few hospitals had difficulty achieving building-level accuracy, as one hospital IT administrator related:

Due to the concrete, we had substantial problems especially in that one building, but we noticed even in newer parts of the facility where we didn't have the concrete walls, that locations would jump, not only between floors or between rooms, but sometimes between buildings. We knew there was a piece of equipment tagged sitting in, for instance room 330 , yet it would show up across a substantial breezeway between buildings in surgery, really unexplainable.

Often the systems could identify the location of inventory and people in a way that should be "close enough" for a person to see, should she or he go to that location, except that the ad hoc design of many hospitals interferes. One biomedical engineer explained:

Nothing's pinpoint [with RTLS], but it gets you within an eyeshot. The only thing is that building design can be a problem, and that's because some of the buildings, the way that they're designed, where [the receiver] may have to plug in will be on an interior wall of a long exterior wall. The unit may be on the other side of that wall, and still being read as

\footnotetext{
3 Another way that nurses attract the ire of administrators is by maintaining on hand any equipment that they need frequently. Administrators find this inefficient and disparagingly refer to the practice as "hoarding." By placing location tags on mobile equipment that needs to be shared on hospital floors or between departments, administrators seek to rationalize equipment use, minimize duplicate items, and reduce time spent searching for needed inventory. Elsewhere we have noted how this rationalization process is usually a top-down exercise that attempts to replace an existing social system of equipment sharing with a technical system of administrative control (Fisher and Monahan 2008).

4 In addition to the categories of "performance" and "behavior," Priscilla Regan (1998) has identified a third category of workplace surveillance, which is "personal characteristics," such as genetic predispositions. Surveillance within this third category was not evident in our research.
} 
where the receiver is. And so, you may go into a room and go, "Well, it's not in here," and then go outside and look on the hallway, and look on the hallway on the other side, but you forget that you've got a 30-foot wall to walk around either side to see if it's on the other side within 7 -feet of that.

Ultrasound systems, which utilize sound instead of radio waves, do not have the same kinds of problems of signals bleeding through walls. But hospitals' spaces do not rely solely on walls to separate patients or zones. Emergency departments, for instance, may use curtains that do not stop sound waves, and this can generate inaccuracies too.

The lack of accuracy understandably frustrates users of the systems to the point where they develop a cynical distrust of the information conveyed and often refuse to use the systems. Many staff members who thought that RTLS was good enough to assist them with finding equipment complained that determining an item's location was insufficient: they also needed to know whether the item was being used and - if not - whether it was clean. In some cases, staff could make assumptions that if portable equipment was in a room with a patient then these items were in use - and some locational software even automates those "associations" - yet ultimately someone must go and look to be certain. The systems are just not "smart" enough to reduce that labor. As a technological fix to this problem, some companies include "in use" buttons on the equipment tags. Vendors report, however, that hospital personnel are not compliant with this process and will rarely push the buttons to indicate the status of a machine.

Another problem associated with RTLS tags is that they carry infection risks when patients are tracked using them, particularly if they are reused on different patients. One hospital IT person told us:

One of the other things we experienced early on, we wanted to tag our emergency department patients and we wanted to be able to do that, but due to the turnover in the emergency department, using a single-use tag was [financially] prohibitive, so we wanted to use a multi-use tag, 2-year battery life. The problem we ran into was you can't submerse them, you can't clean them, you can't sterilize them. So we actually, we ran a few tags through on single patients and sent them to the lab to be analyzed after wiping them down, and found that there were some issues. There were some, you know, some body fluids that get in the cracks.

One somewhat questionable response to this problem of not being able to reuse patient tags is to have a disposable shell but keep the electronic insides intact. It is not entirely clear that the problem of tenacious "body fluids" is eliminated with this solution. Some companies have offered a different technical solution of designing RTLS tags that can withstand the heat of a sterilizing autoclave procedure. Such tags are necessarily more expensive. Users of these autoclavable tags complain that the transmitters in the tags power down during the sterilizing procedure and while cooling, meaning that they are temporarily invisible and thus lost to the system. It is also uncertain how many times a piece of battery-powered electronic equipment, like an RTLS tag, can withstand being autoclaved.

Another significant technical constraint is the need to monitor and replace batteries in "active" tags. While battery monitoring and replacement may seem trivial, when one is talking about potentially tens of thousands of tags with batteries, the labor and cost can be prohibitive. This is especially the case when systems do not reliably alert staff to tags with low batteries, which was a common complaint. With most configurations we saw, battery life for tags was about two to four years, ${ }^{5}$ depending on how often the tags "ping" the detectors to communicate their unique MAC address and location. If the tag is programmed to

5 Vendors whom we interviewed claim their tags have a much longer battery life, but hospital sites with those vendors' equipment have different stories to tell. 
ping less often, say every 10 minutes, then the battery life will increase, but "real-time" accuracy will not be achieved - battery life and locational accuracy are a zero-sum game. ${ }^{6}$

Finally, there is a general lack of interoperability among hospital information systems, and this can deter adoption of systems like RTLS that may be viewed as non-essential. ${ }^{7}$ The lack of interoperability, and even the lack of standardization, can be thought of as being intentional. A hospital technology consultant explained the reasons behind this:

[With HITs] the really big players have business models that are dependent on them having proprietary advantage... One of the biggest things we're fighting is this vendor proprietary advantage issue... And it's just a big battle, and any CIO knows it, they want vendors, they want technologies to interoperate better, but there are technological limitations and just institutional advantages for the vendors not to play nice.

Absent any standards-setting body for hospital IT or any regulation of vendors, technology companies have no incentive to "play nice" and make their systems work with other hospital hardware or software, whether databases, networks, or interfaces. The result may be systems that intensify labor for RTLS users and distract them from their care-related tasks, as one nurse confessed:

Just the fact that we have a lot of different systems, you know? That's not a benefit... you have to work hard to make the system work for you and then I think the other thing that's frustrating is [that] these systems are supposed to make our care more efficient and right now that's not how we're feeling.

When RTLS are perceived by front-line workers as interfering with health care delivery, which is of course not the case for everyone, then support and adoption will remain low and surveillance functions thereby attenuated. ${ }^{8}$

\section{Material Constraints}

The success or failure of technologies must always be understood in relation to the contexts within which they operate. An important contextual dimension of hospitals is the space itself, the unique materialities and histories of buildings, the infrastructures that assert practical orders, and built forms that entrain human emotions, relations, and identities (Andrews and Kitchin 2005; Lindsay 1988; Martin 2000). Hospitals can date back hundreds of years, with countless renovations that become obscured or entirely forgotten over time. Older buildings present special challenges for infrastructure alteration, ranging from electrical insufficiencies, to impermeable firewalls and lead-lined rooms, to dangerous asbestos insulation. These challenges can be amplified by the need to maintain a sterile environment for hospital patients.

\footnotetext{
6 This can be the case as well with tags that are programmed to send out signals only when they are moving. If they do not often move, then long battery life and the potential for accuracy can coexist, but if they do move frequently, which is the presumption upon which RTLS is based, then the batteries will deplete rapidly.

7 A related category of technical concerns and constraints with RTLS has to do with potential health risks introduced by the systems. A 2008 study published in the Journal of the American Medical Association reported, for instance, that RFID systems had been shown to induce electromagnetic interference in medical devices, which could cause serious medical complications and endanger patients (van der Togt et al. 2008). In reference to that study, informants told us that they had witnessed radio frequency signals interfering with the settings of certain patient ventilators that did not have sufficient shielding against radio waves.

${ }^{8}$ Based on our site visits to hospitals, we found a much higher level of satisfaction with and use of RTLS when the systems were utilized for inventory-tracking alone and operated exclusively by "materials management" and/or "biomedical engineering" departments that are not directly involved in patient care.
} 
Wiring hospitals for RTLS detectors is no easy matter. A few buildings afford additions of this sort with interstitial spaces between floors, where workers can introduce more network wires to the already teeming snakes of cable. Most sites are not so accommodating. Once one does wire and install RTLS detectors, power them, connect them to existing or new servers, and so on, then the RF, ultrasound, or other signals can be inconstant because of different building materials. A technologist at an older hospital related:

Okay, so you saw that it's a hodgepodge of build-on situations where we started with a building from 1928 and then we built on again in the 70s and we built on again in the 90s and so you've got different building code construction. So that changes the variability in those RFID readers to pick up those, those actual radio waves.

A frequent story we heard was that IT personnel and engineers never suspected that there were so many lead-shielded rooms at their hospital sites until they solved the mystery of why signals (from tags) would inexplicably vanish in certain parts of the hospital:

If you go into older buildings, there's often some buildings that have lead lining and it's not just where you think they are. Like for example in imaging suites, in the cath labs [catheterization laboratories] come to mind. But there's often other rooms that are leadlined as well because that's where they usually, if they deliver isotope type of treatments that has to be in a lead-lined room and it took us a little bit to find out where those are because we have two rooms in this hospital that most people didn't know about, so again I had to go back to somebody that has been here since the building opened that remembered, to get us that information because otherwise things [items with RTLS tags] go in there, they're lost.

It is often the case that spaces resist effective RTLS implementation in these unexpected ways, such that they frustrate aspirations to systematically track devices or people.

Building design also shapes individual practices and the flow of bodies through space. Because administrators and technologists do not share the same vantage point of nurses, patient transporters, or other front-line staff, they often make wrong assumptions about how people and things move through hospitals, and this leads to design oversights on their part. The following story from a biomedical engineer offers a telling illustration of how different positionalities create blind spots for engineers implementing RTLS:

We were wiring the place and you know being an engineer you think you can figure out everything, right? So we kind of looked at the schematics of the building, we laid out about you know, for going to the cath lab we wanted to just do portal activity because that's where a lot of our pacers go. So I thought I covered all the entryways. So I'm like you know this is all the entryways on this. You know, I know the hospital pretty well; I've been here for a while, so I said let me ask one of the transporters. So I'm walking down the pike [and] I see Fred. ${ }^{9}$ I say, "Hey Fred do you have a second?" He said, "Sure James." I said, "You know can I show you this plan we're doing?" He looked at it [and] he said to me, "James, very, very smart but that's not the way we go." And I'm like, "Well how do you mean?" He said, "James at night that's not the way I go because I have to quickly send the patient down there, rush back upstairs to take another patient. Let me show you where we go." And he proceeded to take me where, I was like okay, egg all over my face, right? So he walks down to this back alley and that's where they take

\footnotetext{
${ }^{9}$ Pseudonyms are used in all quotes to preserve the confidentiality of interviewees.
} 
patients. So we had to kind of quickly go back and rewire for this back alleyway because that's where things would come in and come out without us capturing it.

There is a compulsion to track inventory or people in every conceivable hospital location, but that is more difficult than it seems. RTLS tags frequently fall off, disappear with devices down laundry or trash chutes, move through back hallways and freight elevators, leave through the morgue when patients die, or depart the facility for reasons other than theft, such as patients going outside to smoke or patients being transported to other medical facilities while on tagged stretchers, in tagged wheelchairs, or hooked up to tagged IV stands. Hospitals are fluid, mutable, and porous environments that defy the aspirations of total control that might align better with more sealed and secured spaces.

\section{Financial Constraints}

Just as global economic crises have the potential to slow the "surge" of surveillance and security commitments in police and intelligence sectors (Murakami Wood 2009), so too do financial constraints hinder the widespread or full-scale adoption of hospital RTLS. With hospitals, however, pressing economic difficulties are constant at most facilities, and the dual missions of patient care and financial solvency leave little room for expenditures on systems not proven to further one or both of those missions. The costs can be extreme too. The hard-wired components of systems can run $\$ 50,000$ to $\$ 100,000$ for each hospital department, and hospitals tend to have many departments, so the cost may be a million dollars or more. Inventory tags containing batteries can run $\$ 50$ to $\$ 70$ per tag, potentially for thousands of items, although some tags are significantly less than this. Basic disposable tags for patients are around $\$ 9$ a piece, which will be a recurring expense for hospitals, some of which see thousands of patients a month. Then, of course, many of the systems do not come with software to run them, or the software is inadequate for the needs of hospitals, so that must be purchased and implemented separately. Additionally, hardware and software require constant maintenance and troubleshooting, even if a site is able to get it up and running satisfactorily and convince staff and others to use it.

With the burden of cost and the questionable efficacy of RTLS, hospital administrators and others can have a difficult time convincing their organizations to purchase these systems. This is especially the case when other needs are perceived as being more pressing or more likely to provide a "return on investment" (ROI). One physician framed it this way: "Maybe it would be nice [to have these systems], but it all has to cost tons of money, you know, and sometimes it's like maybe that money might be better spent on who knows, basic services for people. Or more staff because the nurses are so overloaded." And when it comes to solvency, most hospital administrators believe they can discern quite clearly what investments will be sure bets. An administrator at a large urban hospital reflected:

The [RTLS] technology is there. The cost, though, is also there. And there are many places that want to have the technology but can't afford to have technology or don't think they can afford it. Or they have a choice: Either we do technology or we build a new wing where we have more capacity. They usually choose the capacity because the capacity brings them more money. Buying new technology does not bring in more money. Buying new technology brings in efficiency, which in your ROI, return on investment, you hope brings in more finances.

It can be the case that hospitals decide to implement RTLS in a top-down manner, usually under the direction of technology-oriented CEOs or CIOs, who were referred to by our informants as "visionaries." 10 More often, though, hospital departments operate somewhat independently in their pursuit

\footnotetext{
10 A common strategy used by RTLS vendors is to offer to "pilot" their system in a hospital or a hospital department either "free of charge" or at a discounted rate. This serves a dual purpose of getting the vendor's foot in the door at a given hospital and
} 
of new technological systems. They can strike up relationships with vendors and petition for the hospital to offer financial support for RTLS, but departments are their own, often atomized, organizational entities that oversee their own budgets and are managed by their own chairpersons or directors. This can introduce difficulties when departments want to advocate for hospital-wide systems. A business director of a large hospital related:

Our clinical engineering supervisor here tried to make it a mandated thing, every piece of new equipment comes in has a tag on it. We're like, "Great, that's wonderful. We would love to have that. Who's gonna pay for it?" And he's like, "Well, the departments." Well, you can't mandate something that the departments have to pay for [so that system was not implemented].

Hospital administrators can also be judgmental of departments that claim the RTLS are necessary; administrators can see this as a sign that the departments are not being run appropriately. As one proponent for RTLS for inventory tracking explained:

It was just better to spend [money] on more critical things... [With RTLS] you're spending money to solve a problem that really shouldn't be a problem if you're managing correctly and staff are not losing equipment, and things like that. That's what made it a difficult sell [for us]... It's almost like you're throwing money at a problem that can be managed or should be able to be managed in a different way that shouldn't cost you any money. And so, it's sometimes a difficult sell because what you want to say is, "Instead of spending $\$ 50,000$ to put in a system for a department, just don't lose your stuff."

Advocates for RTLS are well aware of the difficulties of convincing others to invest in these systems and - aside from technology vendors - are largely sympathetic to the arguments against RTLS in hospitals, as the above quote illustrates. There was a strong sense that these systems might be appealing but ultimately unnecessary or redundant, especially for the tracking of people. One director of an ED at a large urban hospital, for instance, was enthusiastic about new dashboard systems for monitoring patients and their care but did not see how RTLS could improve the efficiency or awareness of his staff:

When you got patients lining the walls, lining everywhere, yelling and screaming, you know, three of them in this room, I don't need an electronic system to tell me I'm up to my ass in alligators, I mean, I kind of know it!

One of the ways in which vendors convince administrators that the systems will deliver ROI is through the automation of billing. As mentioned above, the idea behind this is that if a patient comes in contact with a hospital technician, device, drug, or so on, a software-based dynamic association can be made whereby that person or that person's insurance company is billed for that service. This was described to us as one of the holy grails of hospital-based RTLS. One administrator explained this approach by invoking a compelling television commercial he had seen:

I think the best commercial was about IBM when that gruffy looking guy who was stealing all the food and he walks out and he [the clerk] says, "Oh sorry, you forgot your

cultivating advertising opportunities (i.e., vendors can claim that their systems are being used at well-known and reputable hospitals, and they can identify those hospitals by name in their advertising brochures or sales pitches to other sites). Vendors hope that once some of their hardware and/or software is deployed at a given hospital that that site will turn to the same vendor should they choose to expand the system. This approach can backfire too: one hospital we visited was relocating to an entirely new facility and was planning on working with a different RTLS company for the new building because of their frustration with the piloted system and its company. 
receipt!" That's what I want. All that other stuff, the patient leaves, chink! There's the bill! That'll work!

Unfortunately, at the few sites we visited where such dynamic associations were being made in conjunction with RTLS, the functions simply did not work. Instead, they introduced serious headaches for staff and patients who were unable to alter the billing records to correct mistakes.

As should be apparent by now, the various constraints or complications with the adoption of RTLS in hospitals overlap and reinforce one another. Without proven efficacy or return on investment, most hospitals will have a difficult time justifying the implementation of costly and potentially disruptive systems, particularly in materially and organizationally challenging environments. Moreover, when the systems lend the appearance of surveillance, they are especially prone to encountering resistance on the organizational level, to which we now turn in the next section.

\section{Cultural Constraints}

The organizational cultures of hospitals are powerful agents of social regulation. While they may vary significantly from site to site and country to country, they clearly shape professional roles and identities, modes of interaction, status relationships, and philosophies and practices of care (van der Geest and Finkler 2004; Weinberg 2003). The success of new technologies in hospitals depends in large part on their harmonization with existing organizational cultures. If systems are perceived as infringing upon existing territory, intensifying or complicating work, not serving patient care, or negatively altering power relations, they will likely meet with resistance, either overt or covert, or both (Koppel et al. 2008; Timmons 2003). Hospital real-time location systems are no exception to this rule.

A combination of territoriality, competing agendas, and scarce resources explains typical forms of cultural opposition to RTLS. Sometimes different departments in the same hospital have ties to different technology vendors, or even have partnerships or other financial stakes in the success of certain vendors. For instance, some administrators or physicians may "consult" for vendors or sit on the board of directors for technology companies. It would make sense, therefore, that such hospital personnel would oppose systems that they saw as competing with their own personal or financial interests. At other times resistance occurs because of competing goals, such as materials-management departments wanting to track inventory and clinicians wanting to track patients. Information technology or information science departments also have a stake in the systems being considered; we saw instances where IT personnel insisted on systems using existing WiFi networks, so that their initial labor would be minimized, and instances where they demanded that RTLS use different networks altogether, so that RTLS signals would not compromise existing network functionality. In other cases, nursing staff would remove and not replace patient armband tags if these tags impeded administering IV treatments to patients. There was even one example in which the chairperson of an emergency department ordered the removal of RTLS detectors from the department because he felt that they were not aesthetically pleasing.

Much more colorful forms of resistance included selective noncompliance and outright sabotage on the part of hospital employees. Nurses can engage in what administrators refer to as "workarounds" to circumvent RTLS functions that increase their labor (Koppel et al. 2008). Removing tags from equipment is one way that nurses maintain their own inventory (in closets, behind ceiling tiles, or in plain sight) without coming under scrutiny of those running the RTLS system, presuming that managing inventory with RTLS is not the job of nurses. Similarly, as was mentioned earlier, nursing staff and others sometimes remove tags from patients when discharging them, and this confounds automated bedmanagement systems and reduces nurses' workload. Whether intentional sabotage or not, RTLS detectors are sometimes unplugged and not plugged back in, which skews locational readings even further and 
provides additional discouragement for people to use the systems. Practices of this sort aggravate vendors to the point where they lose their composure, as the following passage shows:

Interviewer: I see the "Do not remove" written right across the top there [on a detector]. I mean, to what extent is that really a problem?

Vendor: So, what we've done on the next version is we would actually put a metal strip here and here [on either side of the detector], and when you grab it to pull it out of the socket, it shocks the crap out of you so you let go.

While this vendor was not serious about designing a technological fix to harm people if they tampered with the equipment, he recognized that the success of his company depended on demonstrating locational reliability and that the everyday tasks of cleaning staff, repair persons, and others threatened the performance and therefore hospital personnel's perceptions of his company's products.

Intentional sabotage occurs too. For instance, in a group interview with staff at a hospital that had a fully functioning system for inventory tracking, they reminisced about the failed attempt to track security staff at the hospital:

They wouldn't wear them [the tags]. Didn't like them. They wouldn't wear them. So you have to decide, what are your policies? Is it a condition of employment? Is it something that you're going to get disciplinary action for? Are you going to track me to the bathroom? Yeah. The system that we're using... because it's in limited production, the costs are pretty high. We're somewhere between 40 and 50 dollars a tag. Do I put out that money and then somebody puts it [the tag] in the flower pot? Which is where I'm finding them, up in the ceiling [and] in the flower pot. They didn't want to be tracked. They thought that every move they made was being tracked... The individual officers stopped wearing their tags one by one. No, they wore their tags. They broke them. They put them in water. They did everything to destroy them and kept them on [to disguise the fact that they were being noncompliant].

We also heard of several instances of nurses destroying both inventory-tracking and staff-tracking RTLS tags. With a few exceptions, physicians would use their higher status positions to simply refuse to wear locational tags. Hospital administrators, it should be noted, were simply never asked to wear tags, at least not in any of the hospitals we studied.

When administrators trying to deploy RTLS meet with such forms of resistance, they tend to conclude that it is simply not worth the effort to implement the systems. This holds true especially when the intention is to track people, but it can also be the case when the objective is to track equipment. In one example, RTLS was implemented in part to take automated temperature readings in refrigerators containing temperaturesensitive substances or materials (e.g., blood, breast milk, body parts, drugs, blankets). We quote at length the following interview passage about the destruction of temperature readers in refrigerators because it communicates the profound sense of frustration that administrators can feel as a result of technology sabotage, which in turn can bring about a sense of apathy on their part:

Administrator: We went to turn it [the RFID system] on and hundreds of devices were ripped out already. So people thought we were listening to them, so they unscrewed the little antennas, they were ripping the probes out of the equipment.

Interviewer: So they saw the antennas and presumed it was audio recording? 
Administrator: Yeah, even though we communicated it, yes, audio, motion tracking... So I think what it probably was, was just a few of the paranoid-type people on each shift that may or may not have been directly involved with that function [of taking temperature readings], just saying, "Oh what's that? Are they listening to you" and then you get somebody talking about a conspiracy theory... So they were like, "You know, they're probably just trying to figure out if they should fire some of us and what we're doing," and, I mean, pretty bad. So we actually, when we go up there, these antennas now are super-glued, screwed in and bolted down... Unless you make it completely invisible and you don't talk about it, staff are going to rip the devices off, even if you put them on the beds. They don't care if they're really never gonna have to look for a bed again or they know when they have to replace the bed, the point is they're attached to the bed and there's some kind of thought process that's saying, "They're gonna know what I'm doing because I'm associated with this bed or this IV pump." It's a complete failure [the RFID system], right, you know? I mean I've sunk $\$ 300,000$ on it... So that's the snapshot of 5 years of pain.

While not many hospital sites gave us such detailed stories about their "failures," many confessed that they anticipated opposition, particularly to the "Big Brother" associations with staff tracking, and decided not to implement those systems or those components of RTLS. An administrator at one university hospital whose nurses belong to a union explained: "No, we can't tag nurses. We are a union facility. We'd have to get union approval to tag staff, same with our techs and everything. That's just a battle we wouldn't want to try." In this case, the culture of the hospital is shaped by explicit workers' rights and collective bargaining agreements that make getting permission to do surveillance of this sort too arduous. Most other hospitals conclude that staff tracking does not have a sufficient clinical goal to justify the effort - it would just be tracking for tracking's sake:

[Nurses] didn't want somebody tracking them and they thought when they went to the restroom or when they went on break [they would be tracked]. And what we tried to say [is], "It's only within certain locations and it's only related [to workflow]." ... We at the end of the day said, "It's really not worth fighting [for], "cause what would we use this data for?"

In a slightly different register, some administrators equate the need for staff tracking with the admission of some sort of personnel or management problem: "You can get a system, a name-based system that tracks your employees, but you know if you're not having a problem with your employees, why do you need to track them?" Framed in this way, RTLS loses its allure because few hospitals or hospital departments would ever want to admit - at least not publicly - that they have problems managing personnel.

Organizational cultures can significantly impede RTLS in hospitals. Some of the forms of resistance covered in this section could be understood as counter-surveillance practices on the part of hospital staff to the data imperatives and control efforts of hospital administrators. As with resistance to surveillance in other domains, tactical efforts by people occupying lower status positions may be more about challenging issues of power asymmetry than with contesting threats to privacy (Gilliom 2001; Monahan 2006). This would certainly better explain the sabotage of RTLS tags on equipment or the circumvention of bedmanagement systems. Because administrators did their best to keep us from front-line users of RTLS systems and because front-line workers were largely suspicious of us as outside researchers, we were unable to document actual saboteurs' rationales for destroying tracking equipment. Similar to the Luddites of $19^{\text {th }}$ century England (Winner 1977), though, hospital employees are certainly not anti-technology: apparently only the systems that are perceived of as threatening or impinging upon the livelihood of nurses or other staff are being dismantled or circumvented. Surveillance-capable systems that introduce 
dissonances with existing, if ever-mutating, organizational cultures, are often deemed simply not worth the trouble of implementing.

\section{Conclusion}

Regardless of domain of study, it is important to document and theorize existing constraints upon and absences of surveillance. Otherwise there is a risk that Surveillance Studies scholars will succumb to - and thereby reify - a type of technological determinism, perhaps imposing this framework upon their data. The Surveillance Studies community is astute at deconstructing myths of technological determinism when police, politicians, media representatives, or technology vendors claim that surveillance and security devices are necessary and inevitable (e.g., Andrejevic 2007; Samatas 2004; Wilson and Sutton 2004). Drawing upon the sociology of scientific knowledge, we would like to suggest that a kind of methodological "symmetry" and "reflexivity" is in order (Bloor 1991), whereby scholars similarly challenge their own assumptions about the surveillance potentials of new technologies. ${ }^{11}$ This is not an attack, not by any means; instead it is more of a confession. In our project on hospital real-time location systems, we were looking for surveillance and convinced that we would find rampant cases of it. There certainly were disturbing instances of surveillance, some with harsh disciplinary actions associated with them, but the totality of our data suggested something perhaps more interesting and inspiring: tenacious obstacles to surveillance.

In this paper we identified four areas of obduracy facing hospital RTLS: technical, material, financial, and cultural. The primary technical constraint is that the systems simply do not work as promised, at least not yet, to reliably track equipment or people in hospitals. Hospital buildings themselves resist alteration on the material level: finite space for new systems limits what can be deployed, and the ways in which people move through these complex spaces are not always intuitive. The cost of RTLS also impedes their widespread deployment, especially given their indirect and sometimes tenuous connection to improved patient care or financial gain. Finally, organizational cultures introduce significant hurdles to the successful use of new systems; these constraints can range from interdepartmental competitiveness, to general non-compliance, to outright sabotage. Obviously these four dimensions are intended to serve as a heuristic for categorization and analysis, but they definitely overlap and reinforce one another. In other research settings, it might be more appropriate to use different and/or additional categories.

In conclusion, we affirm that it is imperative to continue to flag the many surveillance potentials of new technological systems, including their propensity for mission creep, automation, or convergence with other systems. At the same time, we recognize that there are many reasons for systems to fail, and sometimes there is more rigidity than momentum when it comes to surveillance. Thus, the future may not be as dire as much scholarship in the field implies; we may not be moving toward a "society of control" after all, or at least not as rapidly or inexorably as some fear (e.g., O'Harrow 2005; Parenti 2003). What we have done in this paper is try to call attention to some of the forms of friction that surveillance systems may encounter. Generally speaking, resistance to surveillance may find more success if and when it draws upon existing forms of everyday obduracy in specific contexts, be they national, cultural, organizational, or otherwise.

\footnotetext{
11 Technically speaking, under the Strong Programme of the sociology of scientific knowledge (SSK), our mobilization of "symmetry" here is actually a conflation of two SSK tenets: impartiality and symmetry. We are advocating for impartiality with respect to analyses of the success or failure of surveillance-capable systems and symmetry with regard to explanations for why certain systems succeed while others fail. However, we are not suggesting that social science research can be value neutral, which is why taking reflexivity seriously would require attention to one's own politics and interests and to the values and reward structures that govern one's field (Chubin and Restivo 1983; Hess 1997).
} 


\section{Acknowledgments}

This material is based upon work supported by the U.S. National Science Foundation under grant numbers SES-0642797 and SES-0907993.

\section{References}

Andrejevic, Mark. 2007. iSpy: Surveillance and Power in the Interactive Era. Lawrence, KS: University Press of Kansas.

Andrews, Gavin J., and Rob Kitchin. 2005. Geography and Nursing: Convergence in Cyberspace? Nursing Inquiry 12(4): 316324.

Ball, Kirstie. 2005. Organization, Surveillance and the Body: Towards a Politics of Resistance. Organization 12(1): 89-108.

Berg, Marc. 1997. Rationalizing Medical Work: Decision-support Techniques and Medical Practices. Cambridge, MA: MIT Press.

Bijker, Wiebe E., and John Law, eds. 1992. Shaping Technology / Building Society: Studies in Sociotechnical Change. Cambridge, MA: MIT Press.

Bloor, David. 1991. Knowledge and Social Imagery. 2nd ed. Chicago: University of Chicago Press.

Blumenthal, David, and John P. Glaser. 2007. Information Technology Comes to Medicine. New England Journal of Medicine 356(24): 2527-2534.

Bowker, Geoffrey C., and Susan Leigh Star. 1999. Sorting Things Out: Classification and Its Consequences. Cambridge, MA: MIT Press.

Chubin, Daryl E. and Sal Restivo. 1983. The 'Mooting' of Science Studies: Research Programmes and Science Policy. In Science Observed: Perspectives on the Social Studies of Science, edited by K. D. Knorr-Cetina, and David Mulkay. Beverly Hills: Sage, 53-83.

Fisher, Jill A. 2006. Indoor Positioning and Digital Management: Emerging Surveillance Regimes in Hospitals. In Surveillance and Security: Technological Politics and Power in Everyday Life, edited by T. Monahan. New York: Routledge, 77-88.

Fisher, Jill A. and Torin Monahan. 2008. Tracking the Social Dimensions of RFID Systems in Hospitals. International Journal of Medical Informatics 77 (3): 176-183.

2011. The 'Biosecuritization' of Healthcare Delivery: Examples of Post-9/11 Technological Imperatives. Social Science and Medicine 72 (4): 545-552.

Gilliom, John. 2001. Overseers of the Poor: Surveillance, Resistance, and the Limits of Privacy. Chicago: University of Chicago Press.

Hess, David. 1997. Science Studies: An Advanced Introduction. New York: New York University Press.

Holm, Nicholas. 2009. Conspiracy Theorizing Surveillance: Considering Modalities of Paranoia and Conspiracy in Surveillance Studies. Surveillance \& Society 7(1): 36-48.

Koppel, Ross, Tosha Wetterneck, Joel Leon Telles, and Ben-Tzion Karsh. 2008. Workarounds to Barcode Medication Administration Systems: Their Occurrences, Causes, and Threats to Patient Safety. Journal of the American Medical Informatics Association 15: 408-423.

Lindsay, Prior. 1988. The Architecture of the Hospital: A Study of Spatial Organization and Medical Knowledge. The British Journal of Sociology 39(1): 86-113.

Lohr, Steve. 2009. Wal-Mart Plans to Market Digital Health Records System. New York Times, March 11. http://www.nytimes.com/2009/03/11/business/11record.html [accessed January 16, 2011].

Lyon, David. 1994. The Electronic Eye: The Rise of Surveillance Society. Minneapolis: University of Minnesota Press. . 2001. Surveillance Society: Monitoring Everyday Life. Buckingham, England: Open University.

Martin, Colin. 2000. Putting Patients First: Integrating Hospital Design and Care. The Lancet 356: 518.

Mesman, Jessica. 2008. Uncertainty in Medical Innovation: Experienced Pioneers in Neonatal Care. New York: Palgrave Macmillan.

Monahan, Torin. 2006. Counter-surveillance as Political Intervention? Social Semiotics 16(4): 515-534.

. 2010. Surveillance in the Time of Insecurity. New Brunswick: Rutgers University Press.

Monahan, Torin, and Jill A. Fisher. 2008. Scanning the Future of Hospital Radio-Frequency Identification Systems. Hospital Information Technology Europe 1 (1):44-45.

Murakami Wood, David. 2009. A New 'Baroque Arsenal'? Surveillance in a Global Recession. Surveillance \& Society 6(1): 1-2.

Murakami Wood, David (ed.), Kirstie Ball, David Lyon, Clive Norris, and Charles Raab. 2006. A Report on the Surveillance Society. Wilmslow: Office of the Information Commissioner.

O'Harrow, Robert. 2005. No Place to Hide. New York: Free Press.

Oudshoorn, Nelly. 2007. Diagnosis at a Distance: The Invisible Work of Patients and Healthcare Professionals in Cardiac Telemonitoring Technology. Sociology of Health \& Illness 30 (2):272-288

Parenti, Christian. 2003. The Soft Cage: Surveillance in America: From Slavery to the War on Terror. New York: Basic Books.

Regan, Priscilla. 1998. Genetic Testing and Workplace Surveillance: Implications for Privacy. In Surveillance, Computers, and Privacy, edited by D. Lyon and E. Zureik. Minneapolis: University of Minnesota Press, 21-46.

Samatas, Minas. 2004. Surveillance in Greece: From Anticommunist to Consumer Surveillance. New York: Pella Publishing Company.

Staples, William G. 2000. Everyday Surveillance: Vigilance and Visibility in Postmodern Life. Lanham, MD: Rowman \& Littlefield Publishers. 
Thuemmler, Christoph, William Buchanan, Amir Hesam Fekri, and Alistair Lawson. 2009. Radio Frequency Identification (RFID) in Pervasive Healthcare. International Journal of Healthcare Technology and Management 10(1-2): 119-131.

Timmons, Stephen. 2003. A Failed Panopticon: Surveillance of Nursing Practice via New Technology. New Technology, Work and Employment 18(2): 143-153.

van der Geest, Sjaak, and Kaja Finkler. 2004. Hospital Ethnography: Introduction. Social Science and Medicine 59: $1995-2001$. van der Togt, Remko, Erik Jan van Lieshout, Reinout Hensbroek, E. Beinat, J.M. Binnekade, and P.J.M. Bakker. 2008. Electromagnetic Interference From Radio Frequency Identification Inducing Potentially Hazardous Incidents in Critical Care Medical Equipment. Journal of the American Medical Association 299(24): 2884-2890.

Weinberg, Dana Beth. 2003. Code Green: Money-driven Hospitals and the Dismantling of Nursing. Ithaca: Cornell University Press.

Wilson, Dean, and Adam Sutton. 2004. Watched Over or Over-watched? Open Street CCTV in Australia. Australian and New Zealand Journal of Criminology 37(2): 211-230.

Winner, Langdon. 1977. Autonomous Technology: Technics-out-of-control as a Theme in Political Thought. Cambridge, MA: MIT Press.

1986. The Whale and the Reactor: A Search for Limits in an Age of High Technology. Chicago: University of Chicago Press.

Winthereik, Brit Ross, Irma van der Ploeg, and Marc Berg. 2007. The Electronic Patient Record as a Meaningful Audit Tool: Accountability and Autonomy in General Practitioner Work. Science, Technology \& Human Values 32(1): 6-25. 